\title{
Behandlungsstrategien bei Verletzungen von Milz und Leber im Kindesalter
}

\author{
Strategies for Paediatric Spleen and Liver Injuries
}

Autoren

Institute
S. Zundel ${ }^{1}$, J. Lieber ${ }^{1}$, I. Tsiflikas ${ }^{2}$, A.-K. Henk ${ }^{3}$, P. Schmittenbecher ${ }^{3}$

${ }^{1}$ Kinderchirurgie und Kinderurologie, Universitätsklinikum Tübingen, Deutschland

${ }^{2}$ Diagnostische und Interventionelle Radiologie, Universitätsklinikum Tübingen, Deutschland

${ }^{3}$ Kinderchirurgische Klinik, Städtisches Klinikum Karlsruhe, Deutschland

\section{Schlüsselwörter \\ - Bauchtrauma \\ - Kinder \\ konservative Chirurgie \\ - Milzruptur \\ - Leberruptur}

Key words

- abdominal trauma

- children

- non-operative management

- spleen injury

- liver injury

\section{Bibliografie}

Dol http://dx.doi.org/ 10.1055/s-0034-1383373 Zentralbl Chir 2014; 139: 592-599 (c) Georg Thieme Verlag KG Stuttgart - New York . ISSN 0044-409X

Korrespondenzadresse Dr. Sabine Zundel Kinderchirurgie und Kinderurologie

Universitätsklinikum Tübingen

Hoppe-Seyler-Straße 3

72076 Tübingen

Deutschland

Tel.: 0 152/01715915

Fax: 07071/204640

sabine.zundel@

med.uni-tuebingen.de

\section{License terms}

\section{()(1) $\Theta \circledast$}

\section{Zusammenfassung}

$\nabla$

Verletzungen von Milz und Leber im Kindesalter werden durch alterstypische Stürze, Sport- oder Verkehrsunfälle verursacht. Bei isolierten Verletzungen hat sich die nicht operative Therapie (NOM) etabliert und es stehen evidenzbasierte Leitlinien zur Verfügung. Die Kompression der Organe durch die geschlossene Bauchdecke im begrenzten intraabdominellen Raum ist die pathophysiologische Erklärung für das Sistieren der Blutung. Voraussetzung für eine nichtoperative Therapie ist die bildgestützte Klassifikation der Verletzungen nach der Organ Injury Scale (OIS) und ein hämodynamisch stabiler Patient. Die Kreislaufstabilität des Patienten wird - falls notwendig - durch Bluttransfusionen, Volumensubstitution und Katecholamine gewährleistet. Bei Gefäßstielabrissen, kompletten Devaskularisierungen mit fehlendem Perfusionsnachweis oder bei instabilen Patienten - trotz Ausreizung der konservativen Therapiemaßnahmen - ist die notfallmäßige operative Therapie zwingend. Bei dieser hat die organerhaltende Chirurgie für Milz und Leber einen hohen Stellenwert. Insbesondere im Wachstumsalter ist die Milz essenziell für die Ausbildung der immunologischen Kompetenz. Die Leberruptur kann durch Verletzungen der Gallengangstrukturen kompliziert sein, die einer operativen Rekonstruktion bedürfen. Handelt es sich um eine Milz- oder Leberverletzung im Rahmen einer Mehrfachverletzung oder eines Polytraumas, so muss eine individuelle Therapieentscheidung getroffen werden. Hierfür existieren keine evaluierten Therapierichtlinien und die Rate der operativen Maßnahmen steigt. Für alle Verletzungsarten gilt, dass der kindertraumatologisch spezialisierte Chirurg durch seine Kenntnisse über anatomische und altersspezifische Besonderheiten des verletzten Kindes im multidisziplinären Team die besten Therapieergebnisse erzielt.

\section{Abstract \\ $\nabla$}

Traumatic injuries of the spleen and liver are typically caused by age-related falls or sports and traffic accidents. Today, the non-operative management for isolated injuries is established and evidence-based guidelines are available. The intact abdominal wall and the limited space within the peritoneum produce a compression which is the pathophysiological explanation for the limitation of the haemorrhage. Precondition for the non-operative therapy is the radiology-based classification of the injury (organ injury scale) and a haemodynamically stable patient. Haemodynamic stability is, if necessary maintained with blood transfusion, volume substitutes and the administration of catecholamines. In cases of hilar vascular injury and devascularisation or haemodynamic instability of the patient, despite utilisation of the measures mentioned above, urgent operative therapy needs to be performed. Organ sparing surgery is the therapy of choice for both liver and spleen. The spleen is required for the development of a competent immune system in the growing organism. Liver injuries can be further complicated by injury to the bile system, which might require operative reconstruction. If a patient suffers from multiple injuries and spleen or liver are involved, the decision on the management needs to be taken individually, no guidelines exist but the rate for operative therapy increases. Independent of the dimensions of injury, an experienced paediatric surgeon with his multidisciplinary team, considering the anatomic and age specific characteristics of a child, achieves the best therapeutic results. 


\section{Einleitung}

Unfälle sind die häufigste Todesursache im Kindesalter. Mitursächlich für letale Verläufe können abdominelle Organverletzungen sein, die bei 2-5\% aller Unfälle im Kindesalter vorkommen. Unfallursachen für die - in Mitteleuropa nahezu immer stumpfen Bauchtraumata - sind alterstypische Stürze, Sportoder Verkehrsunfälle. Als typischer Unfallmechanismus muss die Lenkerverletzung bei roller- oder fahrradfahrenden Kindern hervorgehoben werden. Aufgrund der sicherheitsrelevanten Weiterentwicklung des Materials nimmt die Prävalenz dieses Unfallmechanismus kontinuierlich ab, stellt jedoch mit ca. $20 \%$ nach wie vor die häufigste isolierte Unfallursache dar. Bei unklaren Unfallmechanismen müssen nichtakzidentelle Verletzungen im Rahmen von Misshandlungen ausgeschlossen werden, bei denen in $8 \%$ das Abdomen beteiligt ist [1]. Das Alter der durch Misshandlung intraabdominell verletzten Kinder wird mit 2,5-3,7 Jahren deutlich niedriger angegeben als das der übrigen unfallverletzten Kinder [2].

Die am häufigsten verletzten intraabdominellen Organe sind Milz, Leber und Niere mit je ca. 30\% [3]. Anatomische Besonderheiten des wachsenden Kindes machen Milz und Leber verletzungsanfälliger als beim Erwachsenen. Die Organe sind aufgrund ihrer Lage und Größe weniger durch die Rippen geschützt, häufig besteht ein geringeres Fettpolster und weniger umgebende Muskulatur. Aufgrund des höheren Flüssigkeitsgehalts kommt es früher zum Bersten der Organe. Hinzu kommt, dass Vorerkrankungen für eine Verletzung prädisponieren können.

Die Behandlung der isolierten Milz- und Leberverletzungen hat in den letzten 2 Jahrzehnten einen grundlegenden Paradigmenwechsel erfahren. Unter evidenzbasierten Behandlungsrichtlinien lässt sich heute ein Großteil der Patienten im Wachstumsalter mit isolierten Milz- und Leberverletzungen konservativ behandeln, die Mortalitätsrate wurde hierdurch erheblich gesenkt [4]. Für die Behandlung von mehrfachverletzten Kindern liegen keine Behandlungsrichtlinien vor, das Vorgehen muss individuell festgelegt werden. Der vorliegende Artikel konzentriert sich auf das diagnostische Vorgehen sowie die Therapie von isolierten Milz- und Leberverletzungen im Kindesalter.

\section{Diagnostik}

$\nabla$

Die primäre Diagnostik bei Verdacht auf Verletzungen von Milz oder Leber richtet sich nach dem Gesamtverletzungsbild. Findet sich ein hämodynamisch stabiler Patient mit umschriebenen Verletzungszeichen, stehen die im Folgenden beschriebenen bildgebenden Verfahren mit den jeweiligen Vor- und Nachteilen sowie entsprechenden Kontroversen zur Verfügung [5]. Unabhängig davon ist es unstrittig, dass bei polytraumatisierten Patienten ein Trauma-Scan (hochauflösende Spiralcomputertomografie) im Rahmen der Notfallversorgung notwendig ist [6,7].

\section{Sonografie}

Die Sonografie wird typischerweise initial als standardisierte „focused abdominal sonography for trauma“ (FAST) durchgeführt und erreicht auch beim Kind Spezifitätswerte bis 99\%, die Sensitivität ist jedoch eingeschränkt. Werte aus veröffentlichten Studien variieren [8-10]: Ein systematischer Review mit Metaanalyse ergab eine Sensitivität von 56,5\% [11]. Dies illustriert, dass auch bei fehlender freier intraabdomineller Flüssigkeit eine Verletzung der parenchymatösen Bauchorgane vorliegen kann. Eine unauffällige FAST stellt damit kein relevantes Entscheidungskriterium über das weitere Vorgehen dar [12]. Mit einer vollständigen sonografischen Untersuchung lässt sich die Sensitivitätsrate auf $>90 \%$ steigern, trotzdem verbleiben falsch-negative Befunde ( $\odot$ Abb. 1 a), die ausschließlich mit der Computertomografie (CT) korrigiert werden können ( $\mathbf{A}$ bb. 1 b). Höhergradige Verletzungen ( Abb. 1 c) weisen typischerweise eine deutliche Echogenitätsanhebung auf und eine Klassifikation analog des CTs kann gelingen ( $\bullet$ Abb. $1 \mathrm{~d})$.

Der Sensitivitätsunterschied zwischen Sonografie und CT könnte sich in naher Zukunft unter Verwendung von Kontrastmitteln in der Ultraschalldiagnostik (CEUS - contrast enhanced ultrasound) egalisieren. Eine aktuelle retrospektive Studie an einem gemischt pädiatrischen/erwachsenen Kollektiv konnte eine Sensitivität von 99\% aufzeigen [13]. Aktuell ist die Verwendung des Kontrastmittels bei Kindern noch „off label“, die Forderung nach zeitnahen weiteren Studien und einer „Zusammenarbeit für eine schnelle Anwendung in der klinischen Praxis“ werden jedoch ge-
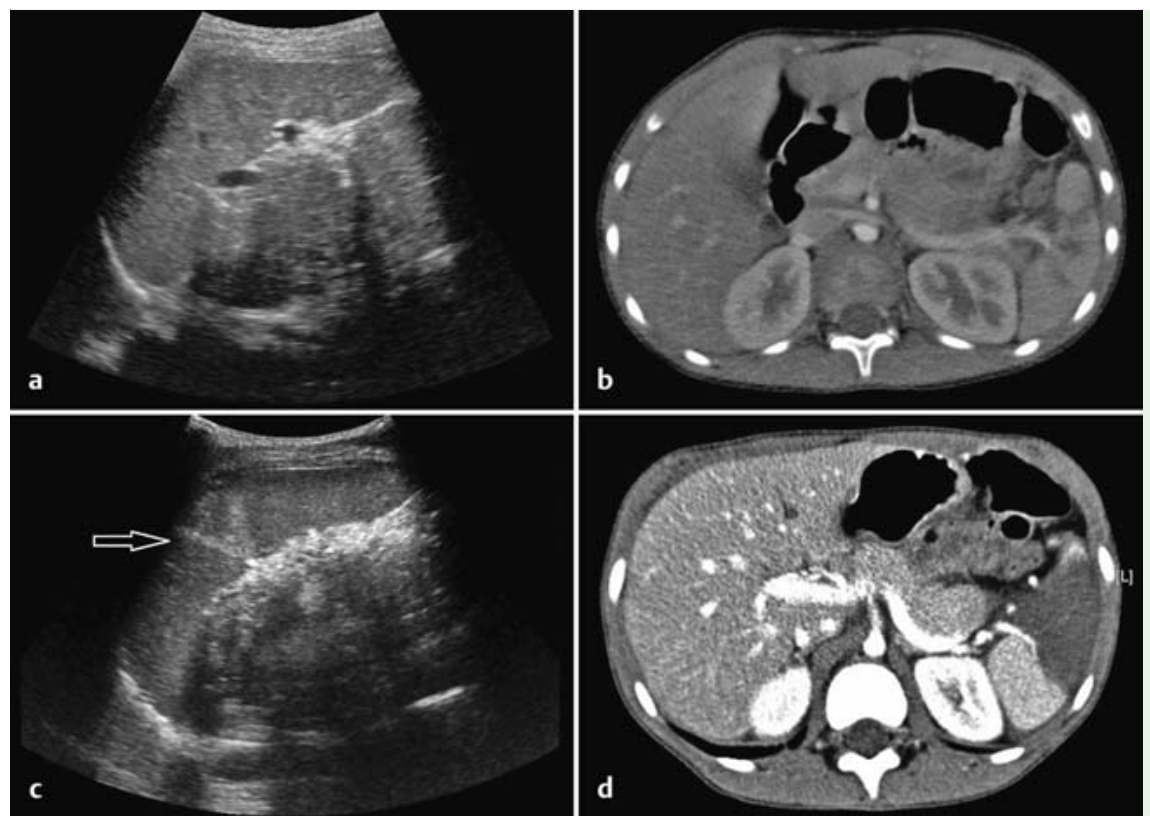

Abb. 1 a bis d Patient A mit zweitgradiger Milzverletzung nach Sturz von einem Klettergerüst. In der Sonografie (a) zeigte sich ein homogenes Milzparenchym, die Verletzung kommt nicht zur Darstellung, es konnte jedoch freie Flüssigkeit mit einzelnen Binnenechos dargestellt werden, worauf die Schnittbildgebung (b) indiziert wurde, bei der die zweitgradige Milzläsion diagnostiziert wurde. Patient B wurde nach Treppensturz mit der Grad-IVLäsion vorgestellt. In der Sonografie (c) zeigten sich im Milzhilusbereich eine keilförmige Echogenitätsanhebung sowie reichlich freie Flüssigkeit mit Binnenechos. Im CT (d) kam der Perfusionsausfall von ca. einem Drittel des Milzparenchyms und das ausgedehnte Hämatom zur Darstellung. 
Tab. 1 Klassifikation der Milzverletzungen nach der American Association for the Surgery of Trauma.

\begin{tabular}{|c|c|}
\hline Grad & Verletzungsmuster \\
\hline I & $\begin{array}{l}\text { subkapsuläres Hämatom: nicht zunehmend, }<10 \% \text { der Oberfläche } \\
\text { betreffend } \\
\text { Lazeration: Kapseleinriss, nicht blutend, }<1 \mathrm{~cm} \text { tief }\end{array}$ \\
\hline II & $\begin{array}{l}\text { subkapsuläres Hämatom: nicht zunehmend, } 10-50 \% \text { der Oberflä- } \\
\text { che betreffend, intraparenchymatös, }<2 \mathrm{~cm} \text { Durchmesser } \\
\text { Lazeration: Kapseleinriss, blutend, } 1-3 \mathrm{~cm} \text { tief ohne Verletzung von } \\
\text { Trabekelgefäßen }\end{array}$ \\
\hline III & $\begin{array}{l}\text { subkapsuläres Hämatom: zunehmend, > } 50 \% \text { der Oberfläche be- } \\
\text { treffend, aktiv blutend oder intraparenchymatöses Hämatom, } \\
>2 \mathrm{~cm} \text { Durchmesser } \\
\text { Lazeration: > } 3 \mathrm{~cm} \text { tief oder mit Verletzung von Trabekelgefäßen }\end{array}$ \\
\hline IV & $\begin{array}{l}\text { Hämatom: intraparenchymale Ruptur, aktiv blutend } \\
\text { Lazeration: segmentale oder hiläre Gefäßdestruktion mit aus- } \\
\text { gedehnter Devaskularisation ( }>25 \% \text { der Milz) }\end{array}$ \\
\hline V & $\begin{array}{l}\text { Lazeration: vollständige Destruktion der Milz } \\
\text { Gefäße: Verletzung der Hilusgefäße mit Devaskularisation der Milz }\end{array}$ \\
\hline
\end{tabular}

äußert [14]. Voraussetzung für eine alleinige sonografische Diagnostik bleibt jedoch die präzise Klassifikation der Verletzung (s.u.).

\section{Computertomografie}

Die CT erreicht bei Leber- und Milzverletzungen Sensitivitätswerte von nahezu 100\% [15]. Mit dieser Untersuchung lassen sich subkapsuläre Hämatome, parenchymale Blutungen und Gefäßbeteiligungen sicher differenzieren. Zusätzlich können die intraund extrahepatischen Gallenwege der Leber beurteilt werden. Der entscheidende Vorteil der CT liegt in der kurzen Untersuchungsdauer von nur wenigen Minuten und in der exakten Bestimmung des Verletzungsgrads ( $\boldsymbol{O}$ Tab. $\mathbf{1}$ und $\mathbf{2}$ ) sowie der damit verbundenen therapeutischen Schlussfolgerungen. Die Indikation zur CT muss dennoch kritisch gestellt werden, da Kinder aufgrund des höheren Entartungsrisikos pro Dosis Strahlung generell einem höheren Risiko unterliegen [16,17]. In diesem Zusammenhang zeigen Daten aus mehreren pädiatrischen Traumazentren der USA, in denen die CT typischerweise zur Basisdiagnostik beim Abdominaltrauma zählt [18], eine hohe Rate (bis zu 75\%) an Normalbefunden [5]. Hershkovitz et al. fordern daher, dass CTUntersuchungen auf Patienten mit passender Unfallanamnese und symptomatischen Befunden beschränkt werden [19].

Idealerweise sollten CT-Untersuchungen bei Kindern mit gewichtsadaptierten Low-Dose-Protokollen angefertigt werden, um eine maximale Dosisreduktion zu erzielen [20,21]. Hierbei finden heutzutage monophasische Kontrastmittel-MultisliceSpiral-CT-Untersuchungen Anwendung, die durch eine initial langsame Kontrastmittelinfusion zur Aufsättigung charakterisiert sind, gefolgt von einer 2. schnellen Kontrastmittelgabe. Damit können die verschiedenen Gewebearten gleichermaßen kontrastiert und gleichzeitig Gefäßstrukturen in einer einzigen Untersuchungssequenz abgebildet werden. Trotz Dosismodulationsprogrammen ist die Strahlenbelastung ca. 100-300-mal höher als bei einer konventionellen Röntgen-Abdomen-Untersuchung.

\section{Labordiagnostik}

Der entscheidende Laborparameter in der Notfalldiagnostik ist das Hämoglobin. Neben dem Vorteil seiner einfachen Bestimmbarkeit spielt er als Verlaufsparameter eine bedeutende Rolle in der Therapie. Der Hämatokritwert lässt Rückschlüsse auf Ver-
Tab. 2 Klassifikation der Leberverletzungen nach der American Association for the Surgery of Trauma.

\begin{tabular}{|c|c|}
\hline Grad & Verletzungsmuster \\
\hline I & $\begin{array}{l}\text { subkapsuläres Hämatom: stationär, }<10 \% \text { der Oberfläche betroffen } \\
\text { Lazeration: Kapseldefekt, stationär, nicht blutend, }<1 \text { cm Paren- } \\
\text { chymtiefe }\end{array}$ \\
\hline II & $\begin{array}{l}\text { subkapsuläres Hämatom: stationär, intraparenchymatös, } 10-50 \% \\
\text { der Oberfläche betroffen, }<2 \mathrm{~cm} \text { Durchmesser } \\
\text { Lazeration: Kapseldefekt, blutend, } 1-3 \mathrm{~cm} \text { Parenchymtiefe, } \\
2-10 \mathrm{~cm} \text { Länge }\end{array}$ \\
\hline III & $\begin{array}{l}\text { subkapsuläres Hämatom: }>50 \% \text { der Oberfläche betroffen oder } \\
\text { rupturiertes subkapsuläres Hämatom, aktiv blutend, intraparen- } \\
\text { chymatöses Hämatom }>2 \mathrm{~cm} \text { oder rasch zunehmend } \\
\text { Lazeration: }>3 \mathrm{~cm} \text { Parenchymtiefe }\end{array}$ \\
\hline IV & $\begin{array}{l}\text { Hämatom: rupturiertes intraparenchymatöses, frisch blutendes } \\
\text { Hämatom } \\
\text { Lazeration: Parenchymriss } 25-50 \% \text { eines Leberlappens }\end{array}$ \\
\hline V & $\begin{array}{l}\text { Lazeration: Parenchymriss > } 50 \% \text { eines Leberlappens } \\
\text { vaskulär: juxtahepatische Venenverletzung der retrohepatischen } \\
\text { V. cava inferior oder großer Lebervenen }\end{array}$ \\
\hline VI & vaskulär: Leberzerreißung \\
\hline
\end{tabular}

dünnungseffekte zu. Als spezifische Parameter gelten die Leberenzyme Glutamat-Oxalacetat-Transaminase (GOT) und Glutamat-Pyruvat-Transaminase (GPT). Ihre Erhöhung auf Werte $>400 \mathrm{U} / \mathrm{l}$ für die GOT und > 250 U/l für die GPT korrelieren mit der Verletzungsschwere der Leber, die bei den angegebenen Grenzwerten auch bildmorphologisch in der CT zu finden ist [22]. Somit kann die Labordiagnostik bei der Indikationsstellung zur CT helfen, darüber hinaus dienen die Transaminasen als wichtiger Verlaufsparameter. Weitere zu bestimmende Parameter sind direktes und indirektes Bilirubin, Gamma-GlutamylTransferase (GGT) und alkalische Phosphatase (AP). Für die Milz gibt es keine spezifischen Parameter.

\section{Weitere diagnostische Verfahren}

Die Magnetresonanztomografie (MRT) ist aufgrund des hohen Zeitaufwands und der Notwendigkeit einer Narkose, um Bewegungsartefakte zu reduzieren, in der Notfallsituation problematisch [23]. Sie kann jedoch sekundär in ausgewählten Fällen zur Klärung von spezifischen Fragestellungen zum Einsatz kommen. So eignet sich die Magnetresonanz-Cholangiopankreatikografie (MRCP) zur Darstellung der Gallenwege bei Verdacht auf Verletzung derselben. Unter Verwendung eines hochspezifischen MRKontrastmittels (Primovist ${ }^{\circledR}$, Dinatriumgadoxetat, Bayer Vital GmbH, Leverkusen), welches zu 30\% von den Hepatozyten aufgenommen und zur Hälfte biliär ausgeschieden wird, ist eine noch bessere Abgrenzung der Gallenstrukturen im Vergleich zur herkömmlichen MRCP möglich [24]. Neben den rein bildgebenden Verfahren bietet die endoskopisch-retrograde Cholangiopankreatikografie (ERCP) zusätzlich die Möglichkeit interventioneller Eingriffe inklusive des Stentings verletzter Gangstrukturen. Im Kindesalter ist hierfür allerdings eine Narkose obligat und die Verfügbarkeit von kindgerechten Geräten ist in vielen Häusern begrenzt.

Die diagnostische Peritoneallavage ist den bildgebenden Verfahren schon durch die Tatsache unterlegen, dass sie eine Verletzung nicht direkt einem Organ zuordnen kann. Weiter ist sie invasiv, benötigt eine Narkose und besitzt ein Verletzungsrisiko für intraabdominelle Strukturen. Zuletzt ist sie nicht entscheidend für das therapeutische Vorgehen und findet deshalb heute praktisch keinerlei Anwendung mehr. Gleiches trifft für die diagnostische 
Laparoskopie zu, die in den bestehenden Kompressionsmechanismus der intakten Bauchdecke eingreift und damit die Grundlage einer konservativen Therapie zerstört. Das Etablieren einer laparoskopischen Übersicht auf ein verletztes Organ während einer hämodynamisch relevanten Blutung ist zudem technisch nur schwer vorstellbar. Dennoch favorisieren einige Autoren die explorative Laparoskopie bei hämodynamisch stabilen Patienten [25] und sehen auch therapeutische Interventionsmöglichkeiten [26], die allerdings im Konflikt zu den evidenzbasierten Behandlungsrichtlinien hämodynamisch stabiler Patienten stehen. Somit dürfte die Laparoskopie beim Kind mit adominellen Verletzungen nur speziellen Situationen wie der Darm- oder Pankreasruptur oder im Einzelfall bei penetrierenden Verletzungen vorbehalten sein.

\section{Nicht operatives Management bei Milz- und Leberverletzungen}

$\nabla$

Die Therapie von Milz- und Leberverletzungen im Kindesalter hat einen grundlegenden Paradigmenwechsel erfahren. So erkannte Wansborough aus dem Kinderkrankenhaus Toronto bereits in den 1940er-Jahren, dass eine Milzverletzung keine absolute Indikation zur Splenektomie darstellt und eine intraabdominelle Blutung bei geschlossener Bauchdecke durch Selbstkompression sistieren kann. Die Erstbeschreibung einer erfolgreichen konservativen Therapie von Milzrupturen bei 12 Kindern erfolgte im Jahre 1968 von Simpson [27]. Über 4 Jahrzehnte hat sich seither ein gravierender Therapiewandel vollzogen und den Begriff des „non-operative management“ (NOM) nunmehr für alle verletzten parenchymatösen Bauchorgane geprägt. Für Milz- und Leberverletzungen erschienen zahlreiche Publikationen mit steigenden Prozentzahlen für konservative Behandlungen, am deutlichsten in den designierten amerikanischen „Kindertraumazentren “ $[28,29]$. Evidenzbasierte Daten lieferten schlussendlich Stylianos et al. 2000 [30]. Die Autoren evaluierten zunächst in einer Multicenterstudie den Ist-Status der Behandlung. Aus der Analyse der Ergebnisse etablierten sie Behandlungsrichtlinien, deren Wirksamkeit sie im Weiteren beweisen konnten [31]. Dieses in der Kindermedizin etablierte Vorgehen hat inzwischen auch in der Erwachsenentraumatologie Einzug gehalten [32-35].

Grundvoraussetzung für die Entscheidung zur konservativen Therapie ist einerseits eine erhaltene Restperfusion des verletzten Organs und andererseits ein hämodynamisch stabiler Patient. Somit können grundsätzlich nur Milz-/Leberverletzungen Grad I-IV nach der amerikanischen OIS konservativ behandelt werden. Komplette Devaskularisationen oder Gefäßstielabrisse (OIS > IV, - Tab. 1 und 2) müssen operativ versorgt werden. Die hämodynamische Stabilität des Patienten wird durch Infusionstherapie, Katecholamingaben und die Transfusion von Erythrozytenkonzentraten erreicht, bezüglich derer nur Empfehlungen, allerdings keine definierten Leitlinien existieren. Nach diesen Empfehlungen sollte die Transfusionsmenge $25 \mathrm{ml} / \mathrm{kg}$ Körpergewicht innerhalb der ersten 2 Stunden oder $40 \mathrm{ml} / \mathrm{kg}$ Körpergewicht innerhalb der ersten 24 Stunden nicht überschreiten. Blutdruck und Puls sind kontinuierlich, der Hämoglobinwert engmaschig zu kontrollieren. Sonografische Kontrollen, um Verlaufsdynamiken freier intraabdomineller Flüssigkeiten zu beurteilen, sollten posttraumatisch in kurzen Abständen (empfohlen werden 4- bis 6stündliche Abstände) durchgeführt werden. Im weiteren Verlauf sind bildgebende Kontrollen nur bei Änderung der klinischen Symptomatik erforderlich. Abgerundet wird das nichtoperative
Tab.3 Empfohlene Richtlinien zur Ressourcenauslastung bei isolierten Milzund Leberverletzungen bei Kindern [31].

\begin{tabular}{|c|c|c|c|c|}
\hline Verletzungsgrad im CT & I & II & III & IV \\
\hline Tage auf Intensivstation & keiner & keiner & keiner & 1 \\
\hline $\begin{array}{l}\text { Krankenhausaufenthalts- } \\
\text { dauer (Tage) }\end{array}$ & 2 & 3 & 4 & 5 \\
\hline Bildgebung vor Entlassung & keine & keine & keine & keine \\
\hline Bildgebung nach Entlassung & keine & keine & keine & keine \\
\hline Sportkarenz in Wochen & 3 & 4 & 5 & 6 \\
\hline
\end{tabular}

Vorgehen durch Bettruhe und initiale Nahrungskarenz bis zur sicheren Stabilisation des Kreislaufzustands. Stylianos inkludierte in seine Veröffentlichungen von 2002 und 2005 konkrete Empfehlungen ( Tab. 3) zum konservativen Management bez. der Notwendigkeit eines Aufenthalts auf Intensivstation, der Dauer des Krankenhausaufenthalts und des Sportverbots [4,31]. Jüngere prospektive Studien legen nahe, dass die empfohlene Liegedauer und Immobilisation weiter verkürzt werden kann [36]. Inwieweit diese Erfahrungen aus dem amerikanischen Umfeld in Mitteleuropa mit anderen poststationären Gegebenheiten umsetzbar ist, muss noch evaluiert werden.

Ein Parameter für die Erfolgswahrscheinlichkeit der konservativen Therapie existiert bei Kindern nicht. In der Erwachsenenliteratur finden sich Hinweise, dass ein „contrast blush“, eine Ansammlung von Kontrastmittel innerhalb oder in der Umgebung des verletzen Organs, die Wahrscheinlichkeit für das Versagen des NOM erhöht [37]. Bei Kindern konnte sich dieser prognostische Faktor nicht bestätigen $[38,39]$.

Die Komplikationsrate bei der konservativen Therapie von Milzund Leberverletzungen ist niedrig. Bei der gelegentlich auftretenden Superinfektion von Hämatomen ist i.d.R. eine antibiotische Therapie ausreichend. In Einzelfällen ist die CT-gesteuerte Anlage einer Abszessdränage indiziert. Im Falle eines unserer Patienten wurden nach primär komplikationsloser NOM der Milzruptur bei Fieber, Schmerzen und erhöhten Entzündungsparametern $850 \mathrm{ml}$ Sekret dräniert. Des Weiteren ist die Ausbildung von Pseudoaneurysmata bekannt, die Inzidenz ist mit 5,4\% bei der Milz und 1,7\% bei der Leber beschrieben und typischerweise liegen Verletzungen Grad III oder höher vor [40]. Diese Phänomene verursachen i.d.R. keine Beschwerden und fallen in elektiven

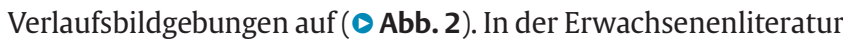
finden sich Berichte von erfolgreichen Embolisationen dieser posttraumatischen Pseudoaneurysmata [41]. Bei Kindern scheint die Rate an Spontanremissionen jedoch höher und die Wahrscheinlichkeit einer Blutungskomplikation niedriger als beim Erwachsenen zu sein, sodass die Notwendigkeit einer elektiven Embolisation infrage gestellt werden muss [42]. Die typischerweise asymptomatischen und selbstlimitierenden Verläufe dieser Bildbefunde haben die Diskussion über den Sinn von bildgebenden Nachuntersuchungen entfacht. Ihre Notwendigkeit wird kontrovers diskutiert $[40,43]$. Zweizeitige bzw. verzögerte Verletzungen, die Bildung von Pseudoaneurysmata sowie die posttraumatische Splenose (Streuung von Milzgewebe mit Implantation in atypischer Lage) sind häufig die Rechtfertigungsgründe für eine Verlaufsbildgebung. Wie oben beschrieben, sind beim Nachweis von Pseudoaneurysmata wie auch bei Splenosen keine therapeutischen Konsequenzen erforderlich. Huebner et al. werteten die Daten von 1083 Kindern aus und fanden keine zweizeitige Ruptur. Sie folgerten, dass die Inzidenz extrem niedrig sei und keine Rechtfertigung für Routinescreenings darstellen [44]. 

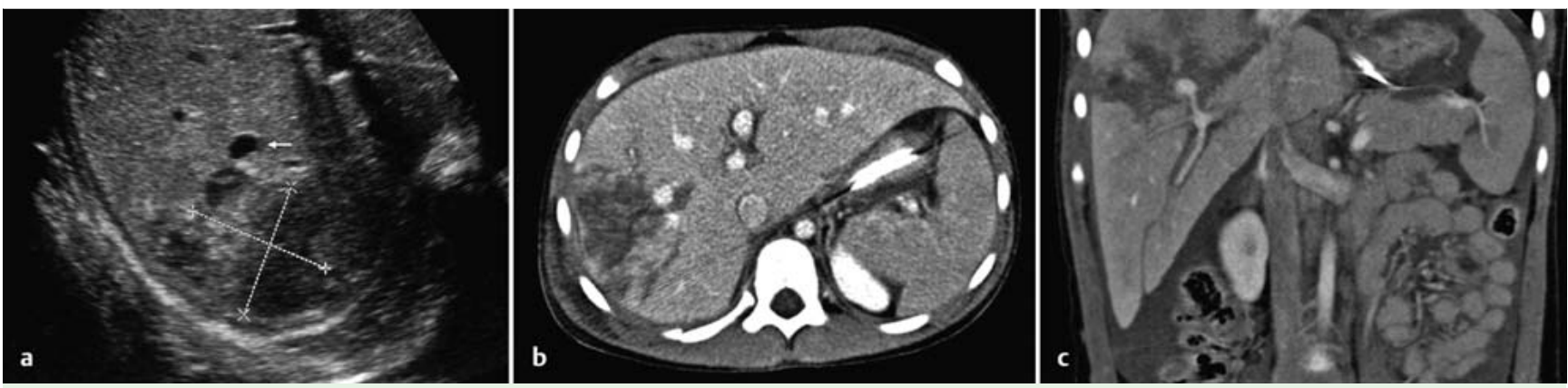

Abb. 2a bis c Die 6-jährige Patientin nach Pferdesturz entwickelte im Rahmen der konservativen Therapie ein Pseudoaneurysma der Lebersegmentarterie VII, welches sowohl sonografisch (a) als auch in der axialen (b) und

Tab. 4 Gemeinsames Patientenkollektiv der Zentren Tübingen und Karlsruhe 2005-2014

\begin{tabular}{|c|c|c|}
\hline & Milzverletzungen & Leberverletzungen \\
\hline Anzahl & 61 & 49 \\
\hline $\begin{array}{l}\text { davon polytraumatisier- } \\
\text { te Patienten }\end{array}$ & 15 & 15 \\
\hline \multicolumn{3}{|l|}{ Unfallursache } \\
\hline Verkehrsunfälle & 16 & 11 \\
\hline Fahrradsturz & 14 & 10 \\
\hline Sturz aus großer Höhe & 10 & 9 \\
\hline Pferdetritt & 2 & 6 \\
\hline andere & 19 & 13 \\
\hline \multicolumn{3}{|l|}{ Organ Injury Scale (OIS) } \\
\hline I & 5 & 4 \\
\hline II & 20 & 12 \\
\hline III & 21 & 18 \\
\hline IV & 15 & 15 \\
\hline $\begin{array}{l}\text { nicht operatives } \\
\text { Management (NOM) }\end{array}$ & $54(88 \%)$ & $46(94 \%)$ \\
\hline Interventionen & $\begin{array}{l}\text { Splenektomie } \mathrm{n}=4 \\
\text { Teilsplenektomie } \\
\mathrm{n}=2 \\
\text { Kapselnaht } \mathrm{n}=1\end{array}$ & $\begin{array}{l}\text { Initial Packing, dann } \\
\text { Blutstillung } \\
\text { Leberoberfläche bei } \\
\text { Second Look } n=2 \\
\text { sekundäre Hemihe- } \\
\text { patektomie bei Rup- } \\
\text { tur des Ductus hepa- } \\
\text { ticus dexter } n=1\end{array}$ \\
\hline Komplikationen & Abszess $n=1$ & $\begin{array}{l}\text { Pseudoaneurysma } \\
n=1\end{array}$ \\
\hline
\end{tabular}

Stylianos schloss sich dieser Empfehlung an und empfahl keine Routinekontrollen nach Krankenhausentlassung (๑ Tab. 3) [31]. Bei Patienten, die posttraumatisch über eine Beschwerdesymptomatik klagen, ist - unabhängig vom Zeitpunkt - eine gezielte Diagnostik durchzuführen.

\section{Operative Therapie}

$\nabla$

Milz

Das Versagen des NOM, bedingt durch die hämodynamische Dekompensation des Patienten, offenbart sich meist innerhalb von 24 Stunden nach Trauma. Im gemeinsamen Patientenkollektiv von Tübingen und Karlsruhe finden sich 61 Patienten mit Milzruptur ( Tab. 4; 2005 bis 2014). Hiervon bedurften 7 Patienten einer operativen Therapie. Die hämodynamische Instabilität zeig- koronaren (c) CT zur Darstellung kommt. Der Befund wurde ausschließlich sonografisch kontrolliert und bildete sich innerhalb von 3 Monaten spontan zurück. te sich bei 1 Patienten bereits im Schockraum, bei 3 Patienten innerhalb der ersten 6 Stunden und bei weiteren 6 Patienten innerhalb der ersten 24 Stunden. Spätere Dekompensationen durch eine zweizeitige Ruptur sind selten. Das häufig beschriebene Phänomen tritt in der Realität aufgrund der stabileren Organkapsel bei Kindern sehr selten auf.

Ist die operative Revision bei einer Milzverletzung unumgänglich, müssen 2 Gefäßversorgungstypen der Milz unterschieden werden: Der Bifurkationstyp kommt mit $84 \%$ am häufigsten vor, der Trifurkationstyp wird mit $16 \%$ angegeben. Es ist zu beachten, dass aus den 2 oder 3 Aufzweigungen der A. lienalis insgesamt 6 Endarterien hervorgehen, die funktionell für die Versorgung eines jeweils zugehörigen Milzsegments verantwortlich sind und somit eine Teilresektion möglich machen. Im Falle zentraler Gefäßligaturen kann die Perfusion durch meist vorhandene Oberpolgefäße oder Gefäße aus dem Omentum minor aufrechterhalten bleiben [3].

Bei oberflächlichen Einrissen - ob solitär oder multipel - ist nach Verfügbarkeit die Anwendung von Nd-YAG- oder Infrarotlasern, Hämostyptika oder Tissucol-Gewebekleber denkbar. Weiter können Kaspelnähte durchgeführt werden oder ein komprimierendes Vicrylnetz („mesh wrapping“) appliziert werden. Bei multiplen tiefen Einrissen sollte über mehrere Segmentresektionen das Belassen einer Restmilz von mindestens 20-30\% der ursprünglichen Organgröße angestrebt werden, um eine ausreichende Milzfunktion zu gewährleisten [3]. Erneut illustrieren die eigenen Daten ( Tab.4), dass situationsbedingt ein Verbluten des Patienten in letzter Instanz nur durch die Organentnahme/Splenektomie als „Ultima Ratio“ zu verhindern ist. Von den o.g. 7 Patienten, die trotz aller Stabilisierungsmaßnahmen hämodynamisch instabil blieben bzw. wurden, konnte eine milzerhaltende Therapie nur in 3 Fällen durch Kapselnaht $(n=1)$ und Teilsplenektomie $(\mathrm{n}=2)$ realisiert werden.

Nach posttraumatischer Splenektomie ist das Risiko eines OPSISyndroms („overwhelming post-splenectomy infection“) 50-fach erhöht, wobei die Gefahr bei den unter 2-jährigen Patienten als nochmals höher eingeschätzt wird [34]. Ein letaler Ausgang dieser durch Haemophilus influenzae, Neisseria meningitides, Pneumokokken oder andere Erreger ausgelösten Sepsiserscheinung wird mit 50-80\% angegeben. $70 \%$ aller OPSI treten innerhalb der ersten 2-3 Jahre nach Splenektomie auf, daher ist eine Pneumokokken-, Meningokokken- und Haemophilusimpfung nach 2-4 Wochen sowie eine orale antibiotische Prophylaxe mit Penicillin V über 3 Jahre obligat [45]. Eine etwaige Autotransplantation von Milzgewebe scheint eine gewisse Erholung der Milzfunktionen 
möglich zu machen, aber weder die Filter- noch die immunologische Funktion kann komplett wiederhergestellt werden [46,47]. Um die Rate an erfolgreich organerhaltend behandelten Patienten weiter zu steigern und somit die immunologische Organfunktion zu erhalten, wurde das Therapiespektrum um die selektive interventionelle Angioembolisation erweitert. In der Erwachsenenmedizin ist die Methode inzwischen verbreitet, die Kriterien für ihre Indikation sind jedoch nicht einheitlich. Sie wird als Alternative zum NOM beschrieben [48], bei Verletzungen > Grad III nach OIS $[49,50]$ sowie bei Pseudoaneurysmata oder AV-Fisteln [51]. Bei Kindern ist das Verfahren beschrieben, die Veröffentlichungen sind Erfahrungsberichte aus einzelnen Kohorten. Auch in diesen Berichten variieren die Vorgehensweisen bez. der Indikationsstellung: Teilweise wurde eine Embolisation primär bei in der CT sichtbarem Kontrastmittelaustritt indiziert [52,53], teilweise erfolgte sie sekundär bei kontinuierlicher Blutung [54, 55]. Technisch erfolgt überwiegend die selektive Embolisation der proximalen Milzarterie. Zur Anwendung kommen sowohl Coils (Tornado coils, VortX platinum coils) als auch resorbierbare Gelatineschwämme (absorbable gelatin sponge torpedoes or slurry). Der Rehabilitationsprozess von embolisierten Patienten unterscheidet sich von rein konservativ behandelten Patienten. Ben-Ishay beschrieb ein Postembolisationssyndrom mit im Verlauf auftretender Leukozytose, Fieber und Bauchschmerzsymptomatik. Die Symptome zeigten sich selbstlimitierend, verlängerten jedoch den Aufenthalt auf Intensivstation sowie im Krankenhaus im Vergleich zu Patienten mit NOM [54]. Die immunologische Funktion nach Embolisation scheint erhalten, sodass keine Immunisierung durchgeführt werden muss $[53,56]$. Zusammenfassend legen die wenigen vorhandenen Daten nahe, dass die Angioembolisation die Erfolgsrate des NOM auch bei Kindern weiter steigern kann. Die Technik sollte Bestandteil einer interdisziplinären Versorgung von pädiatrischen Patienten sein [57].

\section{Leber}

Bei parenchymatösen Leberverletzungen ist die Indikation zur notfallmäßigen operativen Therapie - wie oben beschrieben die hämodynamische Instabilität des Patienten. Grundsätzlich sind die oben beschriebenen operativen Techniken zur Blutstillung auch im Bereich der Leber gültig. Darüber hinaus stellt die primäre chirurgische Rekonstruktion bei Leber- und Gallenwegsverletzungen nicht die Therapie der ersten Wahl dar, da sie oft mit langen Operationszeiten, einem hohen Blutverlust und daraus resultierenden Massentransfusionen einhergeht. Es resultieren Hypothermie, Koagulopathie und Azidose, welche Morbidität und Mortalität deutlich erhöhen. Ziel ist stattdessen die Umsetzung eines multidisziplinären patientenorientierten Therapieplans. Im 1. Schritt („damage control surgery“) wird eine verkürzte explorative Laparotomie durchgeführt, um die massive Parenchymblutung in der Technik des „abdominal packing“ zu stoppen. Lässt sich der Kreislauf des Patienten hiermit stabilisieren, so schließt sich eine 2. Phase der intensivmedizinischen Therapie an. Es wird die Normothermie wiederhergestellt, Gerinnungsfaktoren substituiert und die Sauerstoffsättigung so optimiert, dass eine 2. Operation zur definitiven Rekonstruktion der intraabdominellen Verletzung geplant werden kann. In dieser Phase kann zuvor über den Einsatz der angiografischen Embolisation sowie über endoskopische Verfahren und Stentungen nachgedacht werden. Meist schließt sich die 3. Phase im Sinne einer Second-Look-Operation an, in welcher die definitive chirurgische Rekonstruktion von Gefäß- oder Parenchymverletzungen realisiert wird.

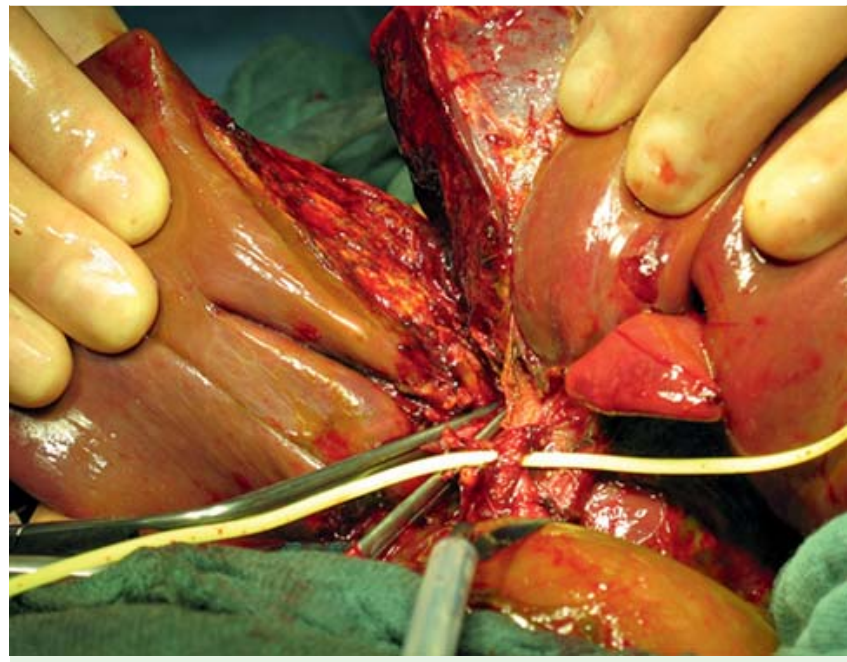

Abb.3 Intraoperatives Bild eines 7-jährigen Jungen nach Lenkertrauma im Rahmen eines Fahrradsturzes. Der Junge war allzeit hämodynamisch stabil gewesen, Bluttransfusionen waren nicht notwendig. In den Kontrollsonografien fiel eine stetig zunehmende Menge an freier Flüssigkeit auf. Zugrundeliegend war die Ruptur des Ductus hepaticus dexter.

Die Leberchirurgie ist aufgrund der arteriellen und portalvenösen Blutversorgung sowie Galleableitung generell höchst anspruchsvoll und inkludiert - auch nach Verletzungen - alle anatomischen Resektionsverfahren sowie vaskulären Rekonstruktionen. Voraussetzung für jede Resektion ist eine vollständige Mobilisation der Leber mit Durchtrennung des Lig. triangulare sinistrum et dextrum, Lig. teres hepatis und Lig. falciforme, um eine ausreichende Übersicht zu gewinnen. Zur Vermeidung eines intraoperativen Verblutens muss das Pringle-Manöver sowie die totale vaskuläre Isolation (Tourniquet der supra- und infrahepatischen V. cava) beherrscht werden. Zuletzt kann der Einsatz der HerzLungen-Maschine notwendig werden, unter welcher eine Organexplantation mit Ex-situ-Rekonstruktion von Gefäßen und nachfolgender Organreplantation durchgeführt werden kann. Ultima Ratio ist die Hepatektomie mit Anlage eines portokavalen Shunts und sekundärer Lebertransplantation [58].

Verletzungen der Gallenblase und der extrahepatischen Gallengänge bedürfen der notfallmäßigen individuellen Therapie, da sie quasi immer im Rahmen von Mehrfachverletzungen mit Leber-, Portalvenen- und/oder Duodenalverletzungen vorkommen. Sie können vereinzelt aber auch beim hämodynamisch stabilen Patienten sekundär durch Zunahme der intraperitonealen freien Flüssigkeit diagnostiziert werden. Gallenblasenperforationen werden mittels Cholezystektomie behandelt. Bei Läsionen der extrahepatischen Gallengänge besteht die Möglichkeit der primären Rekonstruktion oder der Anlage einer biliodigestiven Anastomose. In Einzelfällen kann eine temporäre externe Dränage und Nahrungskarenz zu einem Spontanverschluss eines Leckes aus dem Ductus hepaticus dexter führen [59]. In einem Fall aus dem eigenen Kollektiv konnte eine interventionell-radiologische Stenteinlage eine Leckage nicht abdichten. Bei komplettem Zerbersten des Ductus hepaticus dexter ( Abb. 3) war letztendlich nur eine Hemihepatektomie möglich - wie sie üblicherweise auch bei Rupturen des linken Hauptgallengangs, zentralen intraparenchymatösen Gangverletzungen oder ausgeprägten parenchymatösen Lebergewebezerstörungen indiziert ist. In Erwachsenenkollektiven wird die Inzidenz von therapiebedürftigen Gallenleckagen nach stumpfer Leberverletzung mit ca. $5 \%$ beschrie- 
ben. Hierbei stellt die ERCP die Therapie der Wahl dar, die neben der Stenteinlage eine Papillotomie inkludiert [60]. Bei Kindern berichteten Kulaylat et al. über ein erfolgreiches Stenting in 11 Fällen, allerdings mit gleichzeitiger laparoskopischer Dränageneinlage. Der Gallefluss sistierte nach durchschnittlich 3 Tagen [61].

\section{Zusammenfassung}

$\nabla$

Verletzungen von Milz und Leber im Kindesalter weisen eine hohe Variation in Unfallursachen sowie Verletzungsschwere auf und erfordern einen komplexen diagnostischen und therapeutischen Algorithmus. Die konservative Therapie von Milz- und Leberverletzungen ist etabliert, bei höhergradigen Verletzungen, hämodynamisch instabilen Patienten und bei Vorliegen von Begleitverletzungen ist eine operative Therapie jedoch ggf. notwendig. Die Evaluation, Diagnostik und Therapie von verletzten Kindern sollte interdisziplinär in einem ausgewiesenen kindertraumatologischen Zentrum erfolgen. Die Ergebnisse in einem pädiatrischen Traumazentrum sind nachweislich besser [62-64]. Gestützt wird diese Aussage durch die Tatsache, dass in randomisierten Umfragen Kenntnisse zu den aktuellen evidenzbasierten Therapierichtlinien im Kindesalter in deutlicher Überzahl verneint werden [62]. Innerhalb des pädiatrischen Traumateams muss ein Spezialist für Kinderunfälle zur Verfügung stehen, der die anatomischen Besonderheiten und typischen Unfallmechanismen kennt, der differenziert Diagnostik anweisen kann und der über das operative Spektrum zur Versorgung eines verblutenden Patienten genauso verfügt wie über das der rekonstruktiven Leberchirurgie.

Interessenkonflikt: Nein

\section{Literatur}

1 Roaten JB, Partrick DA, Nydam TL et al. Nonaccidental trauma is a major cause of morbidity and mortality among patients at a regional level 1 pediatric trauma center. J Pediatr Surg 2006; 41: 2013-2015

2 Maguire S, Upadhyaya M, Evans A et al. A systematic review of abusive visceral injuries in childhood-Their range and recognition. Child Abuse Negl 2013; 37: 430-445

3 Lieber J, Fuchs J. Abdominaltrauma. In: Dietz HG, Illing P, Schmittenbecher PP, Hrsg. Praxis der Kinder- und Jugendtraumatologie. Heidelberg: Springer; 2011: 153-179

4 Stylianos S. Outcomes from pediatric solid organ injury: role of standardized care guidelines. Curr Opin Pediatr 2005; 17: 402-406

5 Fenton SJ, Hansen KW, Meyers RL et al. CT scan and the pediatric trauma patient-are we overdoing it? J Pediatr Surg 2004; 39: 1877-1881

6 Jakob H, Wyen H, Marzi I. Polytrauma im Kindesalter. Trauma und Berufskrankheit 2013; 15: 67-74

7 Strohm P, Uhl M, Hauschild O et al. Welchen Stellenwert hat das Ganzkörper-Spiral-CT in der Primärdiagnostik polytraumatisierter Kinder? Z Orthop Unfall 2008; 146: 38-43

8 Coley BD, Mutabagani KH, Martin LC et al. Focused abdominal sonography for trauma (FAST) in children with blunt abdominal trauma. J Trauma 2000; 48: 902-906

9 Luks FI, Lemire A, St-Vil DS et al. Blunt abdominal trauma in children: the practical value of ultrasonography. J Trauma Acute Care Surg 1993; 34: 607-611

10 Patel JC, Tepas JJ 3rd. The efficacy of focused abdominal sonography for trauma (FAST) as a screening tool in the assessment of injured children. J Pediatr Surg 1999; 34: 44-47

11 Schöneberg C, Tampier S, Hussmann B et al. Diagnostik des stumpfen Abdominaltraumas des Kindes - ein systematisches Review mit Metaanalyse. Zentralbl Chir 2013 Aug 1 [Epub ahead of print]

12 Scaife ER, Rollins MD, Barnhart DC et al. The role of focused abdominal sonography for trauma (FAST) in pediatric trauma evaluation. J Pediatr Surg 2013; 48: 1377-1383
13 Sessa B, Trinci M, Ianniello $S$ et al. Blunt abdominal trauma: role of contrast-enhanced ultrasound (CEUS) in the detection and staging of abdominal traumatic lesions compared to US and CE-MDCT. Radiol Med 2014 Jun 25 [Epub ahead of print]

14 Sellars ME, Deganello A, Sidhu PS. Paediatric contrast-enhanced ultrasound (CEUS): a technique that requires co-operation for rapid implementation into clinical practice. Ultraschall Med 2014; 35: 203-206

15 Poletti PA, Mirvis SE, Shanmuganathan K et al. CT criteria for management of blunt liver trauma: correlation with angiographic and surgical findings 1. Radiology 2000; 216: 418-427

16 Brenner DJ, Elliston CD, Hall EJ et al. Estimated risks of radiation-induced fatal cancer from pediatric CT. AJR Am J Roentgenol 2001; 176: 289-296

17 von Wickede M. Diagnostik des stumpfen Bauchtraumas bei Kindern und Jugendlichen. Radiologie up2date; 7: 149-173

18 Pärtan G, Pamberger P, Blab E et al. Common tasks and problems in paediatric trauma radiology. Eur J Radiol 2003; 48: 103-124

19 Hershkovitz Y, Zoarets I, Stepansky A et al. Computed tomography is not justified in every pediatric blunt trauma patient with a suspicious mechanism of injury. Am J Emerg Med 2014; 32: 697-699

20 Riccabona M, Lindbichler F. Traumaradiologie beim Kind. Radiologe 2002; 42: 195-209

21 Tsiflikas I, Seeger A, Schaefer JF. Polytrauma-Spiral-CT im Kindes- und Jugendalter: Weil es so wichtig ist! Pädiatrische Praxis 2014; [im Druck]

22 Holmes JF, Sokolove PE, Brant WE et al. Identification of children with intra-abdominal injuries after blunt trauma. Ann Emerg Med 2002; 39: 500-509

23 Griffiths M, Cord-Uday C. Paediatric Trauma. In: King LJ, Wherry D, ed. ABC of Imaging in Trauma. Chichester, Hoboken: Blackwell; 2010: 97

24 Griffin N, Charles-Edwards G, Grant LA. Magnetic resonance cholangiopancreatography: the ABC of MRCP. Insights Imaging 2012; 3: 11-21

25 Feliz A, Shultz B, McKenna C et al. Diagnostic and therapeutic laparoscopy in pediatric abdominal trauma. J Pediatr Surg 2006; 41: 72-77

26 Marwan A, Harmon CM, Georgeson KE et al. Use of laparoscopy in the management of pediatric abdominal trauma. J Trauma Acute Care Surg 2010; 69: 761-764

27 Upadhyaya P, Simpson JS. Splenic trauma in children. Surg Gynecol Obstet 1968; 126: 781-790

28 Frumiento C, Vane DW. Changing patterns of treatment for blunt splenic injuries: an 11-year experience in a rural state. J Pediatr Surg 2000; 35: 985-989

29 Lally K, Rosario V, Mahour G et al. Evolution in the management of splenic injury in children. Surg Gynecol Obstet 1990; 170: 245-248

30 Stylianos $S$. Evidence-based guidelines for resource utilization in children with isolated spleen or liver injury. J Pediatr Surg 2000; 35: 164169

31 Stylianos S. Compliance with evidence-based guidelines in children with isolated spleen or liver injury: a prospective study. J Pediatr Surg 2002; 37: 453-456

32 Requarth J, D'Agostino jr. R, Miller P. Nonoperative management of adult blunt splenic injury with and without splenic artery embolotherapy: a meta-analysis. J Vasc Surg 2012; 55: 886

33 Hurtuk M, Reed RL2nd, Esposito TJ et al. Trauma surgeons practice what they preach: the NTDB story on solid organ injury management. J Trauma 2006; 61: 243-255

34 Galvan DA, Peitzman AB. Failure of nonoperative management of abdominal solid organ injuries. Curr Opin Crit Care 2006; 12: 590-594

35 Schroeppel TJ, Croce MA. Diagnosis and management of blunt abdominal solid organ injury. Curr Opin Crit Care 2007; 13: 399-404

36 St Peter SD, Aguayo P, Juang D et al. Follow up of prospective validation of an abbreviated bedrest protocol in the management of blunt spleen and liver injury in children. J Pediatr Surg 2013; 48: 2437-2441

37 Schurr MJ, Fabian TC, Gavant M et al. Management of blunt splenic trauma: computed tomographic contrast blush predicts failure of nonoperative management. J Trauma 1995; 39: 507-513

38 Cloutier DR, Baird TB, Gormley P et al. Pediatric splenic injuries with a contrast blush: successful nonoperative management without angiography and embolization. J Pediatr Surg 2004; 39: 969-971

39 Lutz N, Mahboubi S, Nance ML et al. The significance of contrast blush on computed tomography in children with splenic injuries. J Pediatr Surg 2004; 39: 491-494

40 Safavi A, Beaudry P, Jamieson D et al. Traumatic pseudoaneurysms of the liver and spleen in children: is routine screening warranted? J Pediatr Surg 2011; 46: 938-941 
41 Davis KA, Fabian TC, Croce MA et al. Improved success in nonoperative management of blunt splenic injuries: embolization of splenic artery pseudoaneurysms. J Trauma 1998; 44: 1008-1015

42 Frumiento C, Sartorelli K, Vane D. Complications of splenic injuries: expansion of the nonoperative theorem. J Pediatr Surg 2000; 35: 788791

43 Goffette PP, Laterre PF. Traumatic injuries: imaging and intervention in post-traumatic complications (delayed intervention). Eur Radiol 2002; 12: 994-1021

44 Huebner S, Reed MH. Analysis of the value of imaging as part of the follow-up of splenic injury in children. Pediatr Radiol 2001; 31: 852-855

45 Eber S, Belohradsky B, Weiß M. Infektionsprophylaxe bei Asplenie. Klinische Pädiatrie 2001; 213: A84-A87

46 Leemans R, Harms G, Rijkers GT et al. Spleen autotransplantation provides restoration of functional splenic lymphoid compartments and improves the humoral immune response to pneumococcal polysaccharide vaccine. Clin Exp Immunol 1999; 117: 596-604

47 Pabst R. Regeneration of autotransplanted splenic fragments: basic immunological and clinical relevance. Clin Exp Immunol 1999; 117: 423 424

48 Olthof DC, van der Vlies CH, Joosse P et al. Consensus strategies for the nonoperative management of patients with blunt splenic injury: a Delphi study. J Trauma Acute Care Surg 2013; 74: 1567-1574

49 Bhullar IS, Frykberg ER, Siragusa D et al. Age does not affect outcomes of nonoperative management of blunt splenic trauma. J Am Coll Surg 2012; 214: 958-964

50 Stassen NA, Bhullar I, Cheng JD et al. Selective nonoperative management of blunt splenic injury: an Eastern Association for the Surgery of Trauma practice management guideline. J Trauma Acute Care Surg 2012; 73 (Suppl. 4): S294-S300

51 van der Vlies $\mathrm{CH}$, van Delden OM, Punt BJ et al. Literature review of the role of ultrasound, computed tomography, and transcatheter arterial embolization for the treatment of traumatic splenic injuries. Cardiovasc Intervent Radiol 2010; 33: 1079-1087

52 Gross JL, Woll NL, Hanson CA et al. Embolization for pediatric blunt splenic injury is an alternative to splenectomy when observation fails. J Trauma 2013; 75: 421-425
53 Skattum J, Naess P, Gaarder C. Non-operative management and immune function after splenic injury. Br J Surg 2012; 99: 59-65

54 Ben-Ishay O, Gutierrez IM, Pennington EC et al. Transarterial embolization in children with blunt splenic injury results in postembolization syndrome: A matched case-control study. J Trauma Acute Care Surg 2012; 73: 1558-1563

55 Kiankhooy A, Sartorelli KH, Vane DW et al. Angiographic embolization is safe and effective therapy for blunt abdominal solid organ injury in children. J Trauma Acute Care Surg 2010; 68: 526-531

56 Malhotra AK, Carter RF, Lebman DA et al. Preservation of splenic immunocompetence after splenic artery angioembolization for blunt splenic injury. J Trauma Acute Care Surg 2010; 69: 1126-1131

57 Schuster T, Leissner G. Selective angioembolization in blunt solid organ injury in children and adolescents: review of recent literature and own experiences. Eur J Pediatr Surg 2013; 23: 454-463

58 Badger S, Barclay R, Campbell $P$ et al. Management of liver trauma. World J Surg 2009; 33: 2522-2537

59 Feichter S, Esslinger P, Schwöble MG. Ausgedehnte Leberruptur mit Verletzung der Gallenwege auch im Kindesalter nicht immer eine Operationsindikation. Schweiz Med Forum 2011; 11: 142-143

60 Yuan KC, Wong YC, Fu CY et al. Screening and management of major bile leak after blunt liver trauma: a retrospective single center study. Scand J Trauma Resusc Emerg Med 2014; 22: 26

61 Kulaylat AN, Stokes AL, Engbrecht BW et al. Traumatic bile leaks from blunt liver injury in children: a multidisciplinary and minimally invasive approach to management. J Pediatr Surg 2014; 49: 424-427

62 Bowman SM, Bulger E, Sharar SR et al. Variability in pediatric splenic injury care: results of a national survey of general surgeons. Arch Surg 2010; $145:$ 1048-1053

63 Stylianos S, Egorova N, Guice KS et al. Variation in treatment of pediatric spleen injury at trauma centers versus nontrauma centers: a call for dissemination of American Pediatric Surgical Association benchmarks and guidelines. J Am Coll Surg 2006; 202: 247-251

64 Vernon DD, Furnival RA, Hansen KW et al. Effect of a pediatric trauma response team on emergency department treatment time and mortality of pediatric trauma victims. Pediatrics 1999; 103: 20-24 